\title{
SINIDAD Y GEOPOLÍTICA EN EL DESARROLLO DE LA ECONOMÍA CULTURAL CHINA Y SU RELACIÓN BILATERAL COMERCIAL CON ESPAÑ $A^{1}$
}

\section{SINITY AND GEOPOLITICS IN THE DEVELOPMENT OF THE CHINESE CULTURAL ECONOMYAND ITSBILATERAL COMMERCIAL RELATIONSHIP WITH SPAIN}

Gabriel Terol Rojo

Coordinador Unidad Docente del Área de Estudios de Asia Oriental, Universidad de Valencia, (España).

E-mail: gabriel.terol@uv.es ORCID: https://orcid.org/0000-0003-1245-2058

1 Este trabajo forma parte del proyecto de investigación I + D, de la convocatoria 2019 del Ministerio de Ciencia e Innovación de España, titulado 'Nuevos desarrollos socioculturales, políticos y económicos de Asia Oriental en el contexto global' (PID2019-107861GB-I00) y dirigido por el Grupo de Investigación InterAsia de la Universidad Autónoma de Barcelona.

Recepción: 01/05/2020 Aceptación: 29/05/2020 Publicación: 24/08/2020

Citación sugerida:

Terol, G. (2020). Sinidad y geopolítica en el desarrollo de la economía cultural china y su relación bilateral comercial con

España. 3C Empresa. Investigación y pensamiento crítico, 9(3), 17-37. https://doi.org/10.17993/3cemp.2020.090343.17-37 


\section{RESUMEN}

Este documento es una reflexión y un diálogo con una selección de literatura especializada sobre el desarrollo de la economía cultural china y las relaciones bilaterales geopolíticas con España de estas desde la perspectiva sínica.

Inicialmente introduce una visión diacrónica del posicionamiento geopolítico de China, de su herencia ideológica y social, y de la sinización en su entorno regional, así como del interés estratégico de su diálogo con Europa y España. Una vez aquí, expone y debate la relación comercial de China y España, focalizándola entorno a lo cultural, para repasar los rasgos culturales de negociación estereotipados y rebatirlos por el tamiz de la sinidad actual.

Lo que sigue es un estudio sincrónico de la economía cultural china, comprometida con la exposición anterior, para concluir en la sinidad que es posible hallar en ella.

Finalmente, se resuelven los intereses del régimen chino de partido-Estado con la emergente economía cultural por su interés en la construcción y consolidación de los ideales del socialismo chino contemporáneo. Y con ello, se concluye con una reflexión del bilateralismo entre China y España centrado en el desarrollo de la economía de la nueva revalorización de la cultura de China y el rol español frente a ello.

\section{PALABRAS CLAVE}

Sinidad, relaciones comerciales, geopolítica, China, relación bilateral España-China. 


\section{ABSTRACT}

This document is a reflection and dialogue with a selection of specialized literature on the development of the Chinese cultural economy and the geopolitical bilateral relations with Spain of these from the sinitic perspective.

Initially, it introduces a diachronic vision of China's geopolitical position, its ideological and social heritage and the sinization in its regional environment, as well as the strategic interest of its dialogue with Europe and Spain. Once here, it exposes and debates the commercial relationship of China and Spain, focusing on its cultural aspect, to review the stereotypical cultural features of negotiation and refute them through the current sinity sieve.

What follows is a synchronous study of the Chinese cultural economy, committed to the above exposition, to conclude on the sinity that can be found in it.

Finally, the interests of the Chinese party-state regime are resolved with the emerging cultural economy due to its interest in the construction and consolidation of the ideals of contemporary Chinese socialism. And with this, it concludes with a reflection of the bilateralism between China and Spain focused on the development on the economy of the new revaluation of the culture of China and the Spanish role facing it.

\section{KEYWORDS}

Sinity, trade relations, geopolitics, China, bilateral Spain-China relationship. 


\section{INTRODUCCIÓN}

Como recordaba Sean Golden (2019), la revolución epistemológica kuhniana entorno a la interpretación de la historia de la ciencia puso de manifiesto, entre otras cuestiones, la objetividad de las teorías científicas a la vez que alarmó de su complicidad con las ideologías dominantes de su época y algo similar sucede cuando tratamos de hablar de China desde cualquier aspecto. Problematizar marcos teóricos y paradigmas desarrollados por Euroamérica, exige el punto de partida de reflexión sobre la certeza de los atributos que la "modernidad" entendió como fijos, universales y eternos. En ese sentido la problematización que enmarca la posmodernidad en la que se encuentra nuestro presente es exigente con sus enfoques y la actualidad de estos los caracteriza por su enriquecedora interdisciplinariedad y contraste para albergar resultados funcionales y pragmáticos. Por ello, pareciera razonable que tratar sobre la economía cultural de China, y extenderla hasta su relación bilateral con España, exige atender no sólo al contexto geopolítico global, sino también a su idiosincrasia distintiva, ello es, la categoría de "sinidad" que alcanza todas sus actuaciones y deliberaciones.

Para ello, es fundamental tener algunas cuestiones presentes: en primer lugar, que la herencia política e ideológica del país asiático es aún digerida social y gubernamentalmente hacia algún lugar todavía no alcanzado. Heredera de la Revolución republicana, del Movimiento de la Nueva Cultura y del Movimiento del 4 de mayo, de 1912, 1915 y 1919, respectivamente; el partido comunista chino transita hacia el sueño republicano de una "nueva democracia" frente a la "democracia liberal" sin lograr su realización (Golden, 2019). Y por ello, no es infrecuente escuchar que la revolución socialista china se encuentra todavía en activo. La cientificidad del marxismo persevera como filosofía útil para el desarrollo del país desde todas sus perspectivas y el legado maoísta de defender un progreso sin olvidar la personalidad e identidad china recuerda que la máxima 中體西用 algo así como "sirviéndose de lo Occidental [técnica y Ciencia, originalmente] fortalecer o mantener [el cuerpo] lo chino" es la clave para entender correctamente la máxima dengista 一国两制, “un país dos sistemas”. Esa esencia que todos los dirigentes chinos han continuado fortaleciendo desde un proteccionismo variable en relación 
a lo que significaba para cada uno, prevale no sólo en todos ellos si no más importante, en la sociedad y en el paradigma sínico en todas sus manifestaciones. La esencia china, paradójicamente, sigue siendo reescrita. Los retos de la Nueva China no son pocos ni fáciles, pero su realización se construye desde estas premisas.

Si se analiza el histórico de las relaciones chinas en su área regional es posible reconocer de qué manera el proceso de sinización empezó en 1990 desde un punto de vista institucional y económico (Suehiro, 2017), pero también social y de identidad de su diáspora, puesto que la resinización desarrollada en las últimas décadas en estos territorios ha permitido dejar atrás la intención expansionista del socialismo chino para ser sustituido por nuevos atributos ideológicos y, principalmente, económicos e inversionistas y mostrar la hibridación que la caracteriza (Hau, 2012). Una muestra de este interés geoestratégico es posible valorarlo con la Tabla 1 que sigue donde analizando la participación en la EXPO anual CHINAASEAN (CAEXPO), iniciativa creada en el 2000 para promover productos y servicios entre China y los países de la Asociación de Naciones del Sureste asiático (ASEAN), se muestran las cifras comparativas de empresas participantes chinas y pertenecientes a esta asociación, 10 en la actualidad, y los contratos que surgieron de esos encuentros.

Tabla 1. Histórico CAEXPO 2014-2016.

\begin{tabular}{|c|c|c|c|c|}
\hline CAEXPO & \multicolumn{2}{|c|}{$\begin{array}{c}\text { Participación } \\
\text { de empresas }\end{array}$} & \multicolumn{2}{|c|}{$\begin{array}{c}\text { Contratos completados } \\
\text { de empresas }\end{array}$} \\
$\begin{array}{c}\text { Edición } \\
\text { anual }\end{array}$ & ASEAN 10 & China & $\begin{array}{c}\text { Empresas } \\
\text { Empllón de USD) }\end{array}$ & $\begin{array}{c}\text { Empresas } \\
\text { extranjeras }\end{array}$ \\
\hline 2004 & 626 & 1749 & 4968 & 5864 \\
\hline 2005 & 696 & 2518 & 5290 & 6124 \\
\hline 2006 & 837 & 2663 & 5850 & 6945 \\
\hline 2007 & 1126 & 2274 & 6150 & 7651 \\
\hline 2008 & 1154 & 2076 & 6364 & 8807 \\
\hline 2009 & 1168 & 2726 & 6440 & 9054 \\
\hline 2010 & 1178 & 3379 & 6690 & 9962 \\
\hline
\end{tabular}




\begin{tabular}{|c|c|c|c|c|}
\hline CAEXPO & \multicolumn{2}{|c|}{$\begin{array}{c}\text { Participación } \\
\text { de empresas }\end{array}$} & \multicolumn{2}{|c|}{$\begin{array}{c}\text { Contratos completados } \\
\text { de empresas } \\
\text { (Unidad: 1 millón de USD) }\end{array}$} \\
$\begin{array}{c}\text { Edición } \\
\text { anual }\end{array}$ & ASEAN 10 & China & $\begin{array}{c}\text { Empresas } \\
\text { extranjeras }\end{array}$ & $\begin{array}{c}\text { Empresas } \\
\text { chinas }\end{array}$ \\
\hline 2011 & 1161 & s.d & 7420 & 11.316 \\
\hline 2012 & 1264 & 3300 & 8204 & 12.708 \\
\hline 2013 & 1294 & s.d & 9056 & 14.538 \\
\hline 2014 & 1259 & 3341 & 9823 & 16.196 \\
\hline 2015 & 1296 & 3304 & s.d & s.d \\
\hline 2016 & s.d & s.d & s.d & s.d \\
\hline TOTAL & $\mathbf{1 3 . 0 5 9}$ & $\mathbf{2 7 . 3 3 0}$ & $\mathbf{7 6 . 2 5 5}$ & $\mathbf{1 0 9 . 1 6 5}$ \\
\hline
\end{tabular}

Fuente: elaboración propia a partir del estudio de Suehiro (2017).

Sería posible también tomar como muestra de estas tendencias, en la mayoría de sus aspectos, su monumental proyecto conocido como "La iniciativa de la Franja y la Ruta" (一带一路), sin duda legatario de estos propósitos. Es importante conocer que esta iniciativa se divide en una versión terrestre, la Silk Road Economic Belt (SREB) y otra marítima Maritime Silk Road (MSR) y que cuenta con el respaldo financiero del nuevo creado Asian Infrastructure Investment Bank (AIIB). Por un lado, hay que considerar que esta apuesta compromete a más de 60 países y a una población de 4’’ mil millones, pero geoestratégicamente no se puede obviar que resulta clave para entender el concepto de sinización en el siglo XXI. La implementación en su totalidad del proyecto reuniría una elevada cantidad de beneficios en la zona y para China. Son reunidos algunos de ellos en la siguiente Tabla 2.

Tabla 2. Beneficios generales del Proyecto "Franja y Ruta".

\begin{tabular}{|c|c|}
\hline $\begin{array}{c}\text { Fomento del comercio mundial } \\
\text { Reducción del tiempo } \\
\text { de transporte de mercancías }\end{array}$ & $9{ }^{\prime} 7 \%$ \\
\hline $\begin{array}{c}\text { Beneficio directo a la población } \\
\text { de la zona en pobreza extrema }\end{array}$ & $12 \%$ \\
\hline $\begin{array}{c}\text { Beneficio directo a la población } \\
\text { de la zona en pobreza moderada }\end{array}$ & 7 '2 millones \\
\hline
\end{tabular}




\begin{tabular}{|c|c|}
\hline $\begin{array}{c}\text { Incremento } \\
\text { del crecimiento económico global }\end{array}$ & 1 décima \\
\hline Beneficio intangible & $\begin{array}{c}\text { Desarrollo sostenible } \\
\text { mejora de la calidad de vida } \\
\text { de la población mundial }\end{array}$ \\
\hline
\end{tabular}

Fuente: elaboración propia a partir del estudio de Sierra $(2019,30)$.

En el marco europeo, la guía de las relaciones sino-europeas es el llamado EU-China 2020 Strategic Agenda for Cooperation del 2013 y es destacable recordar que si bien empezaron desde un reconocimiento de la asimetricidad de las mismas a favor de China, ello se sostuvo sobre la esperanza de situarlo en las cadenas de valor de la economía mundial para conseguir con ello un pluralismo político y, definitiva, un cambio en su régimen político de partido-Estado, considerándolo como un país en desarrollo (Esteban, 2019). Nadie a fecha de hoy defendería esto último y al haberse convertido en el país que acumula el 20\% de la economía mundial, las críticas por mantener estas políticas son crecientes. Más aún si consideramos que este favoritismo no ha conseguido, sino lo todo contrario, debilitar el régimen de partido-Estado. Nuevamente por olvidar la máxima identitaria utilitarista que fundamenta la esencia sínica.

Por su parte, España mantiene una relación con China, comprometida con la dimensión europea, de evidente socio estratégico debiendo esforzarse por aumentar los recursos en esta interacción, fundamentando un conocimiento riguroso de su socio y luchando por equilibrar la asimétrica relación que también sostiene (Esteban, 2018). Como actor esencial para la gobernanza global, China inquieta la tendencia del orden mundial y nadie duda ya de que la está alterando, activa o pasivamente.

Alcanzado este punto es posible focalizar la atención en cuanto a lo cultural y poner fecha al fundamento institucional de estas relaciones bilaterales con el convenio del 7 de abril de 1981. Destacando desde entonces el aumento significativo de la presencia cultural española en China y viceversa. La cooperación educativa pareciera haber encontrado en las lenguas de cada país un atractivo e ilimitado potencial y haciendo caso de los datos publicados por el Unesco Institute of Stadistics (2016) que sitúa al gigante asiático en la cabecera de exportación cultural del mundo con 60 mil millones de dólares frente a los 15’5 mil 
millones en importación cultural, la balanza evidencia un consumo mayor, en relación a lo que el resto del mundo consume de la cultura china, más que notable. El potencial, no obstante, de ambos países en cultura queda patente al situar a China y a España como el segundo y el tercer país del mundo con más Patrimonio de la Humanidad reconocido por la Unesco, pero esto no se encuentra a la par con la bilateralidad entre ambas potencias y tanto a nivel artístico, editorial, literario, cinematográfico, audiovisual y, en general, todo el conjunto de sectores de la industria cultural, es todavía escaso. La Tabla 3 clarifica este ranking comparativo.

Tabla 3. Balance de exportación e importaciones (en millones de USD) de bienes culturales globales de España y de China continental, 2004-2013.

\begin{tabular}{|c|c|c|c|c|}
\hline \multirow{2}{*}{ Período } & \multicolumn{2}{|c|}{ EXPORTACIONES } & \multicolumn{2}{|c|}{ IMPORTACIONES } \\
\hline & China & España & China & España \\
\hline 2004 & $10.481^{\prime} 27$ & $2.140^{\prime} 06$ & $2.133^{\prime} 92$ & $2.529^{\prime} 43$ \\
\hline 2005 & $12.843^{\prime} 9$ & $2.050 ’ 12$ & $2.360^{\prime} 97$ & 2.755 .18 \\
\hline 2006 & $15.710^{\prime} 78$ & $2.032 ' 10$ & $2.605^{\prime} 36$ & $2.728^{\prime} 19$ \\
\hline 2007 & $18.557 ’ 43$ & $2.200,72$ & $3.498^{\prime} 65$ & $3.358^{\prime} 32$ \\
\hline 2008 & $22.474^{\prime} 01$ & $2.255^{\prime} 33$ & $3.483^{\prime} 75$ & $3.427^{\prime} 09$ \\
\hline 2009 & $18.166^{\prime} 76$ & $1.917^{\prime} 08$ & $3.255^{\prime} 35$ & $2.280 ' 85$ \\
\hline 2010 & $24.849^{\prime} 92$ & $1.840 ’ 29$ & $4.150 ’ 45$ & $2.444^{\prime} 07$ \\
\hline 2011 & $37^{\prime} 712^{\prime} 34$ & $2.252 ' 49$ & $4.716^{\prime} 45$ & $2.401^{\prime} 7$ \\
\hline 2012 & $52.604^{\prime} 71$ & $2.114^{\prime} 58$ & $5.341 ' 2$ & $1.949 ' 81$ \\
\hline 2013 & $60.110^{\prime} 68$ & $2.027 ’ 74$ & $5.840^{\prime} 68$ & $1.858^{\prime} 29$ \\
\hline TOTAL & $273.511 ' 8$ & $20.830^{\prime} 51$ & $37.386^{\prime} 68$ & 25.462 '93 \\
\hline
\end{tabular}

Fuente: elaboración propia a partir del estudio de la UNESCO (2016).

Los elementos culturales en los procesos de negociación en China resultan útiles y un esquema analítico (Castro, 2014) y su simplificación, pueden servir para facilitar su asimilación. Sin duda, los conflictos de interese que trascienden toda negociación pueden verse sensiblemente condicionados por la selección de un buen intermediario (zhōngïānrén, 中间人) y el formalismo chino en cuanto a los negocios descansa desde su tradición confuciana en una gran importancia a la jerarquía (shèhui dèngjí, 社会等级). En ese sentido las buenas relaciones entendidas como armonía social (rénjihéxié, 人际和谐) es también un 
factor determinante. Pero quizás, su mentalidad holística (zhèngtìguānniàn, 整体观念) es el factor más determinante para entender reacciones y valoraciones; y tanto su tendencia al ahorro (jiéjiăn, 节俭), la relevancia de la reputación (miànzi, 面子) y la constancia/resistencia (chīkǔnàiláo, 吃苦耐劳) sean también claves. Con todo, la relación bilateral de la empresa cultural con China cuenta con mucha literatura (Ordóñez, 2004; Bueno, Salmador, Li, 2006) para conocer los principales rasgos distintivos de su filosofía comercial y en nuestro presente actual resulta inusitado no sólo el no conocer sino la no aplicación tanto del concepto de relación recíproca donde los favores y las obligaciones mutuas aseguran la durabilidad de esta (guānxi, 关系); como del concepto que fortalece el vínculo afectivo de los socios participativos de una relación, basado en la confianza y en un sólido sentimiento de círculo íntimo (rénqíng, 人情) y del concepto de crédito personal vinculado a la integridad, credibilidad y nivel de confianza de una persona (xìnyòng, 信用). No obstante, lo que en ocasiones no acaba de clarificar la mayoría de esta literatura, cosificando a la sociedad china, es que los principios prácticos de estas costumbres no son perdurables y que, si atendemos a los cambios sustanciales de China, en ocasiones resultan estereotipados. Es importante recordar que la estrategia popular y social de la práctica del guānxì, por ejemplo, es heredera de la escasez de bienes y oportunidades que en los años sesenta y setenta el monopolio del partido-Estado indujo y que los cambios trascendentes de la sociedad china del siglo XXI legitiman un declive en su uso más allá de ámbitos rurales y localistas (Wank, 2004). Tanto el surgimiento como la estabilización de una economía de mercado, aun con ese similar estructuralizado monopolio estatal, consigue que proliferen relaciones comerciales por interés comercial abandonando sentimentalismos o vínculos de afecto. La rentabilidad y el capital priorizan las relaciones y, por ello, el rendimiento de una gestión comercial prevalece sobre otros valores.

El proceso de sinización es de gran importancia puesto que matiza y posibilita la asimilación correcta del proceder chino y de la inquietud que se esconde tras su identidad. Huelga decir que alcanzando cuestiones culturales que deben ser contextualizadas, así como desarrollos económicos y comerciales que también comprende cuestiones ideológicas, tanto la sinización del marxismo maoísta como el eslogan 
dengista de "socialismo con características chinas" son el germen de la contemporaneidad china (Tian, 2019). Imposibilitando pasar por alto que la actualización del marxismo clásico a través de su sinización dirigió la conducta nacional de China por, entre otros pilares, su particular desarrollo del concepto de “dialéctica” (辩证法), con la participación gubernamental en la reforma económica se ha pivotado hacia un neoliberalismo al modo chino donde la posibilidad de una “crítica dura" (痛砭) augura también una sinización del concepto de neoliberalismo también. Con todo, su extensión a cuestiones culturales y al mismo tratamiento de la cultura reorienta y personaliza no sólo su desarrollo y definición sino también su tratamiento económico y gestión.

\section{METODOLOGÍA}

Alcanzado este punto es posible abandonar la perspectiva diacrónica para centrar otra sincrónica. La metodología utilizada una vez hecha la introducción contextual son fuentes chinas y el planteamiento de exposición son cuatro puntos.

Primeramente, se muestra un análisis de los cambios en la cultura china, desde una visión aperturista que alcance la principal, la subcultura y la anticultura, que puede ser eficiente. El trabajo de Zhang (1997) sirve para enmarcar la tendencia cultural, a finales del siglo XX en China, en tres aspectos: la cultura dominante basada en el poder político, germinal a partir del desarrollo de la sociedad china y comprometida y reflejo del desarrollo ideológico; una subcultura subordinada a la primera y de amplia extensión y una anti-cultura de clara oposición a la primera y que alimentándose de la segunda se convertiría en un sólido fenómeno social de la China contemporánea.

El marco de esta convivencia cultural es el contexto social de transición de una economía planificada a otra de mercado. Esto ha producido en China una transformación y una reconstrucción cultural singular y aunque tradicionalmente su cultura se ha catalogado en nacional y mundial, la clasificación mencionada anteriormente es mucho más realista y práctica. En ese marco, la cultura dominante si bien es reconocida como no estática debe entenderse como claramente heredera de ese marxismo adaptado 
y chino característico. En el período de la Revolución cultural los eslóganes contra el capitalismo del tipo de Xìng wú miè zi (兴无灭资) pueden ser un buen ejemplo de esta y muestra del monólogo cultural dominante. Pero cuando esta deja de ser la principal, aun conservando su nivel de dominante, su convivencia con las demás resulta característica y, a su vez, su nuevo rango. Los cambios de desarrollo en la sociedad china han posibilitado la diversidad social y con ello el desarrollo cultural y el florecimiento de la subcultura afín a esta; sirviendo como recurso de impacto y enriqueciendo la estructura unitaria de la dominante al descubrir nuevas metas y valores, nuevos comportamiento y alteraciones de la tradición. Sin duda, ello es una muestra de la complejidad del desarrollo y cambio social que acontece a la China actual. La brecha entre cultura y realidad es cubierta por la subjetividad de las personas y la subcultura fuerza a la flexibilidad y al dinamismo de la tradición cultural actuando de acelerador. Un papel relevante jugó la cultura extranjera de ocio que la sociedad china consumió con apetito y serviría para estimular esta subcultura de trasfondo, pero también la subcultura religiosa ha cambiado el panorama social chino y no se ha librado de activas controversias con la tradición como sucedería en otros países como los EEUU en la década de los sesenta a propósito del movimiento New Age o similares. Por su parte, la anticultura, en ocasiones entendida como una cierta anti-ilustración, ha venido a extenderse en China como un fenómeno de liberación y esperanza frente al encorsetamiento de la cultura dominante sin perder de vista que nació en occidente en la década de los sesenta y que se haría conocida en China en la década de los ochenta como puede comprobarse por los simposios de la Asociación de Investigación de Cultura Contemporánea de Sichuan (四川当代文化研究会) en 1989. Rasgos destacados de la aportación de la anticultura pueden encontrarse tanto en la vestimenta como en la música consumida actualmente y como, a partir de la década de los noventa, fueron rasgos pioneros de un cambio social de modernización que alcanza al presente actual. En definitiva, estas semillas de adaptación cultural son muestra del pluralismo que ha debido coexistir con los comités de partido y con el denominador común cultural chino por antonomasia: el nuevo confucianismo del siglo XXI. 
Por ello, en segundo lugar, es importante valorar que los cambios acontecidos auguran una nueva era en la economía cultural y que en el caso de la versión china propone una expansión conceptual de esta definiéndola como "economía del conocimiento de la cultura humana". No resulta extraño encontrar estos propósitos en trabajos como los de Fu (2007) donde de acuerdo con las normas de la OMC, expone la necesidad de que China priorice el desarrollo y la gestión de su industria cultural desde plataformas integrales abiertas y ajustables. Esto sirve para entender que el marco global del tratamiento cultural en China va a priorizar unas perspectivas muy claras: por un lado, de globalización cultural, pero también por otro de competencias culturales y, novedosamente, de equilibrio ecológico cultural. Todas ellas se enfrentan a una cosmovisión y filosofía cultural en claro proceso de revisionismo; y para todas ellas, la aportación china será determinante. Resulta paradójico, y para los chinos lo es que, en el proceso de globalización por implementar una estrategia cultural, la potencia china sea un país emergente, a pesar de sus cinco mil años de historia; y que a pesar de esto último la influencia de culturas extranjeras sea considerada un elemento de erosión necesaria, aun así, para su propia madurez. En definitiva, el plan gubernamental de China para hacer frente a la marea de la globalización cultural pasa por promover una esencia étnica y cultivar una cultura avanzada y moderna, junto con la necesidad de activar un mejor estatus internacional.

En tercer lugar, la orientación del valor del desarrollo de la industria cultural china ha resultado sintomática del valor que se le ha conferido a la cultura cuantificando, especialmente, a su valor económico (Xie, 2015). Esto es completamente novedoso frente a anteriores tendencias de claro rechazo de todo cuanto recordara a lo antiguo y a lo clásico. No obstante, es importante no olvidar que se apadrinará gubernamentalmente desde el deseo de que sirva para una guía de orientación de los valores centrales del socialismo chino, integrando sus tradiciones más conservadoras y comprometidas con un desarrollo humanístico de claro beneficio social en un sentido confuciano. Por ello, y es una característica singular, la raíz del desarrollo de la industria cultural en China se lleva a cabo con la intención de aunar tradición y modernidad culturalmente. Básicamente compartiendo el juicio jaspersiano de que la naturaleza social 
se expresa en la herencia mientras que la historia se expresa en la tradición. La primera puede ser alterada por el hombre del presente, pero la segunda es imperecedera. Y esta interpretación justifica la nueva hermenéutica cultural china de respeto e interés por su historia, otrora ni admirada ni tolerada.

Los valores centrales socialistas chinos han encontrado un espacio desde donde liderar una difusión de sus valores, de manera que la expansión de la industria cultural en China, como lo sería en EEUU también, se convierte en instrumento de estos principios no siendo esto, de entrada, unilateralmente negativo. Especialmente cuando las propias estrategias del mercado cultural se encuentran comprometidas con sus respectivas culturas y pudiendo extender esto a ideologías dominantes o todo lo contrario en función de múltiples intereses. La tónica supremacista estadounidense cultural encuentra en China un rival a respetar por mercado de consumo y por estrategia de instrumentalización. El denominado "libre intercambio cultural" aparentemente neutro, por ejemplo, se produce sustancialmente en inglés y ello no está exento de interés y de demarcación; frente a otras culturas marginales que son aceptadas pasivamente, pero que cuenta con una caducidad más estrecha. La orientación cualitativa de la industria cultural china es una noción de "humanización" que se ha convertido desde su tradición confuciana en su elemento cultural de mayor exportación. Y ello es, entre otros motivos, porque encaja a la perfección con los valores del nuevo socialismo chino y su estrategia por dejar sobre la mesa internacional un legado genuinamente chino. Obviamente este atributo convertido en ideal es propio de todas las civilizaciones matizando su interpretación, pero sin duda, va a estar presente en todo el desarrollo cultural nacional chino y va a tratar de convertirse en su legado al mundo. En ese sentido, la gubernamentalización de la industria cultural china diseña priorizar sus beneficios sociales no tanto en rendimiento económico sino de valores. Especialmente los afines a esa "humanización".

Por último, con todo es posible resaltar los indicativos de la construcción de un carácter nacional chino contemporáneo vinculado a la expansión de políticas gubernamentales asociadas a la cultura. Para ellos la aplicación del teorema político de Coase, para cuyo estudio y comprensión el trabajo de Parisi (2003) es muy práctico, en estudios concretos del caso chino son muy convenientes (Fan, 2016) porque 
sobre la base de que las personas tienden a buscar opciones óptimas en todos los aspectos de sus vidas para maximizar el bienestar social permite enfocar el caso chino desde una perspectiva diferente. En este marco, el carácter nacional evolucionaría endógenamente junto con el entorno institucional determinado a su vez por los fundamentos económicos, políticos y geográficos. Pero Fan pone en el tablero la noción de "personalidad" en el orden social, entre otros; y la generaliza en el sentido de identidad comunal o carácter nacional. El caso chino es completamente paradigmático en tanto en cuanto, preexiste una noción de personalidad constante, obviamente artificial, pero continua, y tanto por su extensión geográfica como por su historia resulta singular y única en la historia del mundo. Por un lado, tendríamos su longevidad nacional e histórica vinculada a la noción de imperio y de civilización, pero por otro, el ideológico asociado a estos períodos constataría una sociedad caracterizada en una sumisión social que complementaría un estado de derecho sobre el mantenimiento del orden social. A lo largo de estos períodos no es constatable interés alguno ni preocupación por la unidad cultural ni en ningún consenso moral ni ético porque el confucianismo nutriría de sentido todo el espacio social y cultural. De manera que el ser chino entonces, se identificaba con ser confuciano, ello es, seguir sus normas. El refinamiento de la cultura confuciana permitió rediseñar la personalidad china a través de su cultura y ello germinó como un nacionalismo conservador del orden y jerarquía social. No obstante, los múltiple y diversos hechos que acontecen la historia de China, incluso su presente, subrayan la reiteración por conservar el status quo del país. Y ello, sin duda, no es posible de ninguna manera sin alimentar una idea de carácter nacional, de identidad nacional o de personalidad, en palabras de Fan, aparentemente imperecedero.

\section{RESULTADOS}

La condición y característica aperturista de las políticas chinas empezaron por su economía, alcanzaron a su sociedad y cuentan con unas dos décadas de influencia en su cultura, y más concretamente, en el desarrollo de esta y en su interpretación. Precisamente esta última es la que reúne mayor grado de variación y adaptación. 
La contemporaneidad cultural china ha provocado un paisaje cultural donde la convivencia con fenómenos y producciones culturales lejos de la simplicidad de una cultura dominante ha visto la dispersión social de una subcultura y de una anticultura germinante. Y al igual que sucedería en otras sociedades icónicas de un liberalismo económico y social, China ha tenido que digerir y adaptarse a esta pluralidad caracterizada por una actitud controlada pero crítica en última instancia de la dirección del progreso y de este mismo en sí.

Todo ello ha permitido el establecimiento de una industria cultural que ha propiciado una economía cultural interesante para el régimen de partido-Estado chino, el cual ha modificado su actitud frente a él, para sacarle rendimiento ideológico. Tanto su herencia cultural como sus tradiciones culturales se han reinterpretado para aunar fuerzas entorno a la necesidad de servirse ellas como dinamizadoras de una unidad nacional. Y el trasfondo de esta unidad nacional, entendida como personalidad china ha dilatado un legado sínico reformulado la propia categoría de sinidad como identidad china contrastable y contrastada.

Claramente comprometida con valores ideológicos que surgieron del marxismo chino, el socialismo chino que se expande no esconde la necesidad y el compromiso de reformular estas influencias políticas extranjeras y extraer una versión china cualificada. De manera que sobre conceptos universales como el de humanización, se fomenta una fórmula china que se sirve de su adaptación cultural para exportarse como la solución en la convivencia global a la que nos dirigimos.

El cambio de paradigma cultural universal que propone la experiencia sincrética cultural china descansa en su experiencia por sinizar todo lo recibido de fuera, iniciada con las políticas maoístas más afortunadas, continuada por las denguistas y tomadas como válidas por los dirigentes posteriores hasta Xi Jinping.

No obstante, no habría que confundir la apelación a un universal como una defensa contra la heterogeneidad. Todo lo contrario. La propuesta china fundamenta la supervivencia de las particularidades y por ello, y especialmente en el caso chino, es imprescindible en cuanto a lo comercial y económico, 
pero también cultural, no perder de vista el valor de sus peculiaridades: zhōngjiānrén, shèhuì děngjí, zhěngtǐguānniàn, jiéjiǎn, miànzi y/o chīkǔnàiláo; debiendo actualizar al siglo XXI la aplicación del guānxì, el rénqíng y/o el xìnyòng.

\section{CONCLUSIONES}

Desde 2005, el vínculo entre China y España es considerado un patrimonio, como maniobra compartida con otros países y calificada de estrategia global, valorado bilateralmente como "Asociación estratégica Integral”. No obstante, la relación bilateral económica de la cultural de China y España, presuntamente, no obvia los intereses chinos y la asimetricidad de ambas no esconde el utilitarismo que trasciende la aplicación de la sinidad en el desarrollo económico del desarrollo de la cultura en China. El propio ensanchamiento de la sinidad no esconde sus pilares principales siendo estos claramente los de la superviviencia no de un marxismo clásico sino de su moderna interpretación china.

Coincidiendo en que la orientación correcta del desarrollo de la industria cultural es un requisito de cualquier pretensión de modernización, la máxima china es la de instrumentalizar los valores centrales del socialismo chino para servirse de ellos como orientación de los valores de la industria cultural china. En ese propósito la herencia cultural tradicional ya no es un obstáculo, sino que se sitúa en el mismo nivel que los valores ideológicos que propugnan. En definitiva, se trata de una inversión en el desarrollo cultural a través de una industria que la rentabilice y la guíe para extraer de ella apoyo en "la construcción de un socialismo con características chinas" (中国特色社会主义建设的伟大事业中). Este aforismo es determinante en la relación bilateral económica de la cultura entre China y España.

El paradigma para la diplomacia cultural no escapa a las necesidades de su adaptación en el complejo contexto actual de la globalización (Álvarez, 2019) y su objetivo es, sin duda, sensibilizarse al rol de la cultura y su industria. Sin duda es reconocida como una apuesta estratégica de los gobiernos para alcanzar un mejor posicionamiento en una comunidad internacional multilateral, pero también es un 
recurso para el entendimiento y la comunicación entre los estados. Buena prueba de ello en el caso bilateral de China y España son los Institutos Cervantes y los Centros Confucio.

Y si bien los datos más recientes de la relación comercial relacionada con los bienes culturales europeos determinan que Suiza, Reino Unido y los EEUU representan más de la mitad de todo el montante de exportación (Domżalska, 2019), entre el período del 2013 al 2018 aumentaron, resulta destacable mencionar que China continental pasó del séptimo al sexto consumidor. Los datos de importación son notablemente diferentes, reconociendo a China en el principal origen de las importaciones de bienes culturales europeos tanto en 2013, Tabla 4, como en 2018, Tabla 5.

Tabla 4. Principales importadores de bienes culturales europeos 2013.

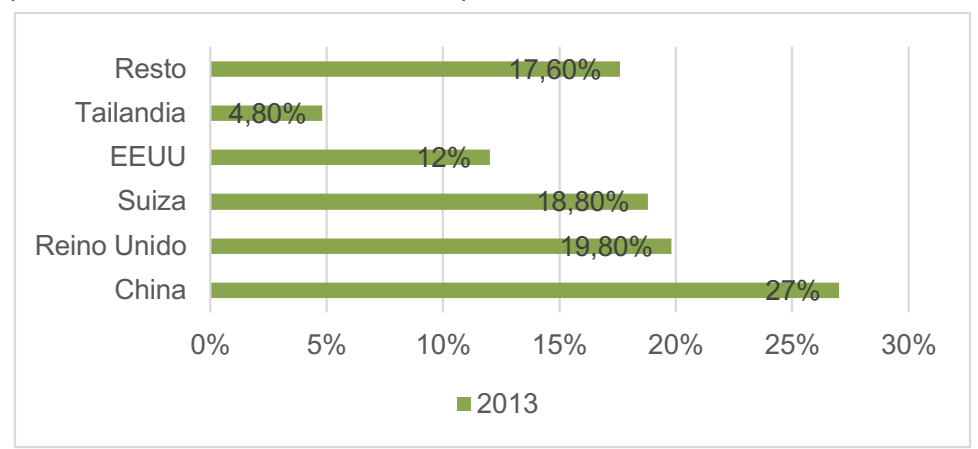

Fuente: elaboración propia a partir del estudio de Domżalska (2019).

Tabla 5. Principales importadores de bienes culturales europeos 2018.

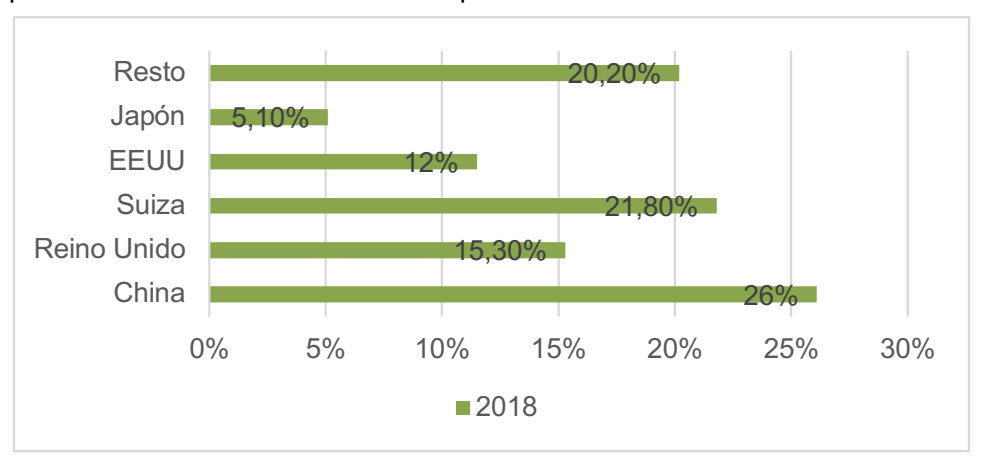

Fuente: elaboración propia a partir del estudio de Domżalska (2019). 
El siglo XXI y las dinámicas de globalización amparadas por la creciente migración supra continental han exigido una nueva valoración de la diplomacia cultural desde donde se nutren las identidades nacionales. China, en este cambio, protagoniza una ofensiva muy sólida, por potencial y por estrategia; pero España, desempeñando un papel insustituible en la portavocía de su lengua y la cultura hispana, también cuenta con potencial y su estrategia no debería quedar descuidada. Y si bien el liderazgo chino en innovación es un hecho (Blanco, 2019), España no debería de desatender la cultura y toda su industria para facilitar más distanciamiento del que hay en la actualidad. Posiblemente, los desafíos que el gobierno chino necesita afrontar para consolidar a su país en innovación sean más accesibles que los relacionados con la cultura.

\section{REFERENCIAS BIBLIOGRÁFICAS}

Álvarez, J. (2019). Hacia un nuevo paradigma para la diplomacia cultural española. ARI 8. Real Instituto Elcano. http://www.realinstitutoelcano.org/wps/portal/rielcano_es/contenido?WCM_GLOBAL_ CONTEXT=/elcano/elcano_es/zonas_es/lengua+y+cultura/ari8-2019-alvarezvalenciahacia-paradigma-diplomacia-cultural-espanola

Blanco, A. (2019). China como líder innovador, entre el éxito y las dudas. ARI 75. Real Instituto Elcano. http://www.realinstitutoelcano.org/wps/portal/rielcano_es/contenido?WCM_GLOBAL_ CONTEXT=/elcano/elcano_es/zonas_es/asia-pacifico/ari75-2019-blancoestevez-china-liderinnovador-entre-exito-dudas

Bueno, E., Salmador, Ma . P., y Li, D. (2006). Guanxi: concepto e implicaciones en la dirección estratégica de las empresas españolas en china. Revista Economía Industrial, 362, 93-101. https:// dialnet.unirioja.es $/$ servlet/articulo? codigo $=2238226$

Castro, O. P. (2014). Elementos culturales en los procesos de negociación internacional. Caso China. Suma de negocios, 5(12), 143-147. https://papers.ssrn.com/sol3/papers.cfm?abstract_id=3019498 
Domżalska, M. (2019). Culture statistics - 2019 edition. Publications Office of the European Union. https://doi.org/10.2785/118217

Esteban, M. (2019). Política europea frente al desafío de China. Comentario Elcano, 7. http://www. realinstitutoelcano.org/wps/portal/rielcano_es/contenido?WCM_GLOBAL_CONTEXT=/ elcano/elcano_es/zonas_es/asia-pacifico/comentario-esteban-oteroiglesias-politica-europeafrente-al-desafio-chino

Esteban, M. (coord). (2018). Relaciones España-China. Informe Elcano 24. http:/ /www.realinstitutoelcano. org/wps/portal/rielcano_es/publicacion?WCM_GLOBAL_CONTEXT=/elcano/elcano_es/ publicaciones/informe-elcano-24-relaciones-espana-china

Fan, C. S. (2016). Culture, Institution, and Development in China: The Economics of National Character. Routledge.

傅守祥 [Fu Shouxiang]. (2007). 文化经济时代: 中国文化产业的发展与管理 [La era de la economía cultural: el desarrollo y la gestión de la industria cultural china]. 深圳大学学报：人文社会科学 版 [Revista de la Universidad de Shenzhen (Sección de Humanidades EO Ciencias Sociales)], 24(2), 134-141.

Golden, S. (2019). China celebra su $70^{\circ}$ aniversario como país «comunista» que defiende el orden liberal frente a países «capitalistas» que pretenden volver al proteccionismo. Observatorio de política china. Entrevistas, 2019/09/11. http://politica-china.org/secciones/china-celebra-su-70o-aniversariocomo-pais-comunista-que-defiende-el-orden-liberal-frente-a-paises-capitalistas-que-pretendenvolver-al-proteccionismo

Bibliografía alternativa de los estudios de Asia Oriental. (2018). Muanfang Magazine. Revista Iberoamericana de Asia Oriental, 5. http://www.yuanfangmagazine.com/ideas/el-experto-recomienda/sean-goldenpropone-una-bibliografia-alternativa-de-los-estudios-de-asia-oriental/

Hau, G. S. (2012). Becoming "chinese" in Southeast Asia. Sinicization and the Rise of China Civilizational, processes beyond East and West. Routledge, 175-206. 
黄少安 [Huang Shao'an]. 经济学研究重心的转移与“合作”经济学构想一对创建“中国经济学”的思 考 [El cambio de enfoque de la investigación económica y el concepto de economía "cooperativa". Reflexiones sobre el establecimiento de la "economía china"], 经济研究 [Investigación económica], $5,60-67$.

Sierra, A. (2019). La influencia de la nueva ruta de la seda en la posición de china en el sistema internacional. un análisis desde las relaciones internacionales (Trabajo Final de Grado). Universidad del País Vasco, España.

Suehiro, A. (2017). China's offensive in Southeast Asia: regional architecture and the process of Sinicization. Fournal of Contemporary East Asia Studies 6(2), 107-131. https://doi.org/10.1080/2476 1028.2017.1391619

Ordoñez, P. (2004). La importancia de guanxi, renqing y xinyong en las relaciones empresariales en China: Implicaciones para las empresas españolas. Tribuna Económica, 818, 221-234. https:// dialnet.unirioja.es/servlet/articulo?codigo $=2327058$

Parisi, F. (2003). Political Coase Theorem. Public Choice, 115(1/2), 1-36. www.jstor.org/stable/30025972

Tian, C. (2019). Mao Zedong, Sinicization of Marxism, and Traditional Chinese Thought Culture. Asian Studies VII(1), 13-36. http://dx.doi.org/10.4312/as.2019.7.1

UNESCO. (2016). The Globalisation of Cultural Trade: A Shift in Consumption. International flowes of cultural goods and services 2004-2013. Unesco Institute of Statistics, París. http://dx.doi.org/10.15220/978-929189-185-6-en

Wank, D. (2004). Business-State Clientelism In China: Decline Or Evolution? Social Connections in China Institutions, Culture, and the Changing Nature of Guanxi. Cambridge University Press, 97-1 15. 
谢传仓 [Xie Chuancang]. (2015). 中国文化产业发展的价值取向 [La orientación del valor del desarrollo de la industria cultural china]. 吉首大学学报(社会科学版) [Revista de la Universidad de fishou (Sección de Ciencias Sociales)], 26(3), 116-122.

张宛丽 [Zhang Wanli]. (1997). 主文化, 亚文化, 反文化与中国文化的变迁 [Cambios en la cultura principal, subcultura, anti-cultura y cultura china]. 社会学研究 [Investigaciones sociológicas], 1, 113117. 